\title{
Measuring rank mobility with variable population size
}

Citation for published version (APA):

Bossert, W., Can, B., \& D'Ambrosio, C. (2016). Measuring rank mobility with variable population size. Social Choice and Welfare, 46(4), 917-931. https://doi.org/10.1007/s00355-015-0942-z

Document status and date:

Published: 01/04/2016

DOI:

10.1007/s00355-015-0942-z

Document Version:

Accepted author manuscript (Peer reviewed / editorial board version)

\section{Please check the document version of this publication:}

- A submitted manuscript is the version of the article upon submission and before peer-review. There can be important differences between the submitted version and the official published version of record.

People interested in the research are advised to contact the author for the final version of the publication, or visit the DOI to the publisher's website.

- The final author version and the galley proof are versions of the publication after peer review.

- The final published version features the final layout of the paper including the volume, issue and page numbers.

Link to publication

\footnotetext{
General rights rights.

- You may freely distribute the URL identifying the publication in the public portal. please follow below link for the End User Agreement:

www.umlib.nl/taverne-license

Take down policy

If you believe that this document breaches copyright please contact us at:

repository@maastrichtuniversity.nl

providing details and we will investigate your claim.
}

Copyright and moral rights for the publications made accessible in the public portal are retained by the authors and/or other copyright owners and it is a condition of accessing publications that users recognise and abide by the legal requirements associated with these

- Users may download and print one copy of any publication from the public portal for the purpose of private study or research.

- You may not further distribute the material or use it for any profit-making activity or commercial gain

If the publication is distributed under the terms of Article $25 \mathrm{fa}$ of the Dutch Copyright Act, indicated by the "Taverne" license above, 


\title{
Measuring rank mobility with variable population size*
}

\author{
WALTER BOSSERT \\ Department of Economics and CIREQ, University of Montreal, Canada \\ walter.bossert@videotron.ca \\ BURAK CAN \\ Department of Economics, Maastricht University, The Netherlands \\ b. can@maastrichtuniversity.nl \\ Conchita D'Ambrosio \\ INSIDE, University of Luxembourg, Luxembourg \\ conchita.dambrosio@uni.lu
}

This version: November 18, 2015

\begin{abstract}
We provide a characterization of a class of rank-mobility measures. These measures generalize the Kemeny measure that is well-known from the literature on measuring the distance between orderings. We use replication invariance to ensure that our measures are applicable in variable-population settings. The rank-based approach to mobility has a natural connection with the study of social status. Rank-based measures are widely applied in empirical research but their theoretical foundation is still in need of further investigation, and we consider our approach to be a contribution towards this objective. Journal of Economic Literature Classification No.: D63.
\end{abstract}

Keywords: Rank mobility, social status, Kemeny measure, variable population.

* We thank Bhaskar Dutta, an associate editor and two referees for their comments. Financial support from the Fonds National de la Recherche Luxembourg, the Fonds de Recherche sur la Société et la Culture of Québec and the Social Sciences and Humanities Research Council of Canada, and the Netherlands Organisation for Scientific Research (NWO) under the grants; i) Open Competitie (OC: 400-09-354) and ii) Innovational Research Incentives Scheme (VENI 2013: 451-13-017) is gratefully acknowledged. 


\section{Introduction}

The measurement of mobility is an increasingly important area within the analysis of social index numbers. The fundamental issue to be addressed is the design of measures that reflect the extent to which members of a society move across social or economic boundaries from one period to the next. A crucial aspect that distinguishes mobility from most other criteria that are used to assess the performance of a society (such as income inequality or poverty) is that mobility is difficult - if not impossible - to define without any reference to intertemporal considerations. Of course, intertemporal approaches to the measurement of inequality, poverty and other social phenomena have been explored but they can also be defined without any difficulties in a single-period setting; in contrast, there is no mobility without movement. As a consequence, the arguments of a mobility measure are pairs of indicators of economic or social status - one indicator for each of the time periods under consideration.

Another characteristic of the concept of mobility is that it is multifaceted. As Fields (2008) summarizes, six mobility concepts can be found in the economics literature: time independence, positional movement, share movement, non-directional income movement, directional income movement, and equalizer of longer-term incomes. Excellent surveys and guides to the literature are also provided by Maasoumi (1998), Fields and Ok (1999) and Jäntti and Jenkins (2014).

In this paper we contribute to the measurement of positional movement or, more specifically, to the measurement of the movement across the ranks held by individuals in a society. The rank-based approach to mobility has a natural connection with the study of social status. Rank-based measures are widely applied in empirical research (see, for example, Dickens, 1999) but, to the best of our knowledge, only few contributions such as D'Agostino and Dardanoni (2009) and Cowell and Flachaire (2011) investigate them from a theoretical perspective. We employ the basic setup of these studies but use different methods and arrive at an alternative class of measures.

D'Agostino and Dardanoni (2009) phrase the problem in terms of (partial) permutation matrices and use a subgroup-consistency property to obtain an additive structure of their criteria. Much of their analysis is devoted to dominance criteria in a fixed-population setting, although they discuss variable-population issues as well without providing formal characterizations. In contrast, we explicitly deal with variable-population considerations by imposing replication invariance and characterizing the corresponding class of rank-mobility measures. An additive structure results in our setting from a standard additivity axiom that is also employed by Kemeny and Snell (1962) and Can and Storcken (2013) in the context of measuring the distance between rankings.

Cowell and Flachaire (2011) propose classes of indices involving various status concepts in a fixed-population setting. Their approach is very flexible and is based on a general measure of distance between individual statuses. The latter may or may not be directly (that is, independently of the position of others) observable. As such, rank mobility is not a central issue in their framework.

In order to perform rank-mobility comparisons across societies with different population sizes, we employ the standard replication-invariance axiom. In our setting, replication 
invariance demands that if a pair of status rankings is replicated, rank mobility remains unchanged. Thus, unlike much of the existing literature on the subject, our measures are applicable in a variable-population framework.

Two dominant measures of non-parametric rank correlation have been established in the literature - namely, Spearman's (1904) $\rho$ index and Kendall's (1938) $\tau$ index. D'Agostino and Dardanoni (2009) characterize rank-mobility preorders that are linked to Spearman's $\rho$ index, whereas we focus on measures based on Kendall's $\tau$ index. We note that Kendall's $\tau$ index and related measures are not included in D'Agostino and Dardanoni's (2009) class. As is the case for all mobility indices that we are aware of, including the rank-mobility measure proposed by D'Agostino and Dardanoni (2009, p. 1796), our measures assume non-negative values only. Clearly, this observation does not apply to the corresponding measures of rank correlation; their values are between minus one and plus one.

The Kendall $\tau$ index is at the core of the Kemeny distance (also referred to as the swap distance), which is one of the most prominent distance measures for orderings; see, for instance, Kemeny (1959) and Kemeny and Snell (1962). By its nature, the rank-mobility setting seems ideally suited for employing the literature on measuring the distance between orderings: the further apart two status rankings are, the higher the mobility inherent in the move from one of these status rankings to the other.

The Kemeny distance is characterized in Kemeny and Snell (1962) but, as pointed out in a recent contribution by Can and Storcken (2013), one of the axioms employed in the original characterization is redundant. As a consequence, Can and Storcken (2013) succeed in obtaining a strengthening of the result due to Kemeny and Snell (1962). The axiom in question is a reducibility condition - the only property used by Kemeny and Snell (1962) that (at least implicitly) links distances between orderings involving different numbers of objects to be ranked.

Although our contribution is concerned with measuring rank mobility rather than the distance between orderings per se, we make use of the results established by Kemeny and Snell (1962) and Can and Storcken (2013). We formulate and discuss axioms that are motivated in terms of the measurement of rank mobility and phrase the most relevant existing characterization in the context of rank-mobility measurement. Using this result as an important intermediate step, we then proceed to our own result - a characterization of a generalized class of rank-mobility indices based on the notion of mobility captured by the Kemeny measure. Variable-population considerations are explicitly taken into account because we employ the well-established replication-invariance principle. To the best of our knowledge, replication invariance has not appeared in the literature on measuring the distance between orderings. This is likely the case because it is a natural property in the context of measurement issues involving the ranking of individuals but is not of immediate appeal in the more abstract setting of measuring the distance between orderings. Thus, the use of this property further distinguishes our approach from the distance-measurement issue and reinforces the conceptual differences between rank mobility and distance measurement.

The next section introduces the basic definitions and the notation used in the paper. A class of rank-mobility measures that generalizes the Kemeny measure is presented and discussed. This is followed by the axioms that we employ in our characterization result, accompanied by a discussion of their suitability in the context of the measurement of rank 
mobility as movements in (social) status. Section 3 is devoted to the statement and proof of our main result, and Section 4 concludes.

\section{Rank mobility}

The notion of using social status as the variable that is relevant for assessing mobility was first analyzed in a theoretical context by D'Agostino and Dardanoni (2009). They employ partial permutation matrices to express movements in the relative ranks of the members of a society and formulate axioms in that setting that allow them to identify a class of rankings of such matrices. While we retain the underlying idea of using changes in relative ranks, we depart from the D'Agostino-Dardanoni framework by using a measure of distance between rankings to assess social mobility. That is, the informational basis - the relative ranks of everyone in society - is the same as in D'Agostino and Dardanoni (2009), but we employ different methods to arrive at what we think of as a reasonable class of mobility measures. The intuition underlying this approach is straightforward: if the distance between the status rankings in two consecutive periods is high, this indicates a high degree of mobility. Throughout, we assume that the status rankings are orderings - that is, reflexive, complete and transitive binary relations - and we refer to the two periods under consideration as period zero and period one.

The individuals are denoted by the set $\mathbb{N} \backslash\{0\}$, while a society $N$ is a nonempty finite subset of $\mathbb{N} \backslash\{0\}$ with a cardinality of at least two. We denote the set of all possible societies by $\mathcal{N}$ and whenever it is clear the cardinality of $N \in \mathcal{N}$ is denoted by $n$. Given a society $N \in \mathcal{N}$, the relative ranks in period zero can be expressed by means of an ordering $R^{0}$ on $N$. Likewise, the relative ranks in period one are given by an ordering $R^{1}$ defined on the same set $N$. Because we want to allow for mobility comparisons across societies with possibly different population sizes, we define a mobility measure as a function that assigns a non-negative value - interpreted as the mobility associated with the move from $R^{0}$ to $R^{1}$ — to any pair of orderings $\left(R^{0}, R^{1}\right)$ that may be defined for any possible population size in $N$. That is, a rank-mobility measure is a function

$$
M: \cup_{N \in \mathcal{N}} \mathcal{R}^{N} \times \mathcal{R}^{N} \rightarrow \mathbb{R}_{+}
$$

where $\mathcal{R}^{N}$ denotes the set of all orderings on $N$.

In this setting, it is natural to borrow from the literature on determining the distance between orderings and one of the most commonly employed measures is what is usually referred to as the Kemeny distance; see Kemeny (1959) and Kemeny and Snell (1962). In the context of this paper, the Kemeny mobility measure $M_{K}$ is defined by letting, for all $N \in \mathcal{N}$ and for all $\left(R^{0}, R^{1}\right) \in \mathcal{R}^{N} \times \mathcal{R}^{N}$,

$$
M_{K}\left(R^{0}, R^{1}\right)=\left|R^{0} \backslash R^{1}\right|+\left|R^{1} \backslash R^{0}\right| .
$$

This definition may appear to be somewhat opaque but it is based on a very intuitive notion of movement from a social-status ranking of yesterday to a social-status ranking today. The Kemeny measure calculates the minimal number of changes one has to apply to an ordering $R^{0}$ to arrive at another ordering $R^{1}$. 
For societies with two agents, e.g., $N=\{1,2\}$, this can be illustrated as follows. There are three possible orderings on the set $\{1,2\}$-namely, $R^{0}=\{(1,1),(1,2),(2,2)\}$, $R^{1}=\{(1,1),(1,2),(2,1),(2,2)\}$ and $R^{2}=\{(1,1),(2,1),(2,2)\}$. Clearly, according to $R^{0}$, individual 1 is ranked higher than individual 2 , in $R^{1}$ the two individuals are ranked at an equal level, and in $R^{2}$ individual 2 is ranked higher than individual 1 . Every pair that is in $R^{0}$ also is in $R^{1}$ and, thus, the set difference $R^{0} \backslash R^{1}$ is empty. Consequently, we have $\left|R^{0} \backslash R^{1}\right|=0$. On the other hand, the only element of $R^{1}$ that is not in $R^{0}$ is the pair $(2,1)$ and we obtain $R^{1} \backslash R^{0}=\{(2,1)\}$, hence $\left|R^{1} \backslash R^{0}\right|=1$. The Kemeny measure applied to these two orderings yields $M_{K}\left(R^{0}, R^{1}\right)=0+1=1$, which is precisely the number of changes necessary in moving from $R^{0}$ to $R^{1}$. Analogously, $M_{K}\left(R^{1}, R^{2}\right)=1$ because $R^{1} \backslash R^{2}=\{(1,2)\}$ and $R^{2} \backslash R^{1}=\emptyset$, thus $\left|R^{1} \backslash R^{2}\right|+\left|R^{2} \backslash R^{1}\right|=1+0=1$. Finally, we obtain $R^{0} \backslash R^{2}=\{(1,2)\}$ and $R^{2} \backslash R^{0}=\{(2,1)\}$ hence $M_{K}\left(R^{0}, R^{2}\right)=\left|R^{0} \backslash R^{2}\right|+\left|R^{2} \backslash R^{0}\right|=1+1=2$. Again, this is the minimal number of changes necessary to move from $R^{0}$ to $R^{2}$, confirming the intuitive interpretation of the Kemeny measure. See Figure 1 for an illustration of the twoindividual case, where the numbers indicated along the lines joining the orderings are the corresponding values of the Kemeny mobility measure. We use the convention of writing, for example, 12 for the ordering such that 1 is ranked higher than 2 and (12) when 1 and 2 are ranked equally high.

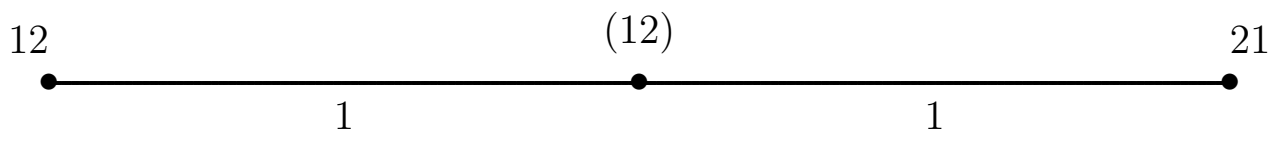

Figure 1: The Kemeny measure for $n=2$

For societies with three agents, e.g., $N=\{1,2,3\}$, there are 13 possible orderings defined on $\{1,2,3\}$. Again, we follow our convention of writing, for instance, 2(13) for the ordering that has individual 2 at the highest status level, followed by 1 and 3 at a lower level, where 1 and 3 are ranked equally high. The Kemeny measure for $n=3$ is illustrated in Figure 2 . The minimal distances are indicated along the lines joining the different orderings.

To obtain the requisite rank-mobility values according to the Kemeny measure, we consider the minimal number of changes needed to reach one ordering from another. For instance, $M_{K}(123,(13) 2)=3$ because three elementary changes are required to move from 123 to (13)2. In this case, there is a unique shortest path (via 1(23) and 132) - any other path from one of these orderings to the other involves more than three elementary changes. In contrast, if we consider the two orderings 123 and 321, any shortest path between these orderings involves six elementary changes and, thus, $M_{K}(123,321)=6$. Now there is no unique minimal path: it is possible, for example, to move from 123 to 321 via 1(23), 132, (13)2, 312 and 3(12), or to use the orderings (12)3, (123) and (23)1 as intermediate steps. In each case, there are six elementary changes involved, and these two paths are not the only shortest paths, as can be verified easily.

Note that, for example, the distance between (123) and 2(13) is equal to two without any other ordering lying in between the two. This is because two elementary changes are 
required to move from one of these orderings to the other and, while there do exist relations that are only one elementary change away from (123), these are not orderings. Thus, the necessity of assigning a distance of two between two neighboring relations is due to the assumption that we restrict attention to orderings.



Figure 2: The Kemeny measure for $n=3$

For societies with more than three members, a diagrammatic representation of all possible orderings defined on the set $N$ appears to be rather cumbersome. For any $N \in \mathcal{N}$, the number of possible orderings on $N$ is given by the corresponding ordered Bell number $B(n)$; see, for instance, http://en.wikipedia.org/wiki/Ordered_Bell_number. The ordered Bell numbers can be expressed recursively by means of the function $B: \mathbb{N} \cup\{0\} \rightarrow \mathbb{N}$ defined by letting $B(0)=1$ and, for all $n \in \mathbb{N} \backslash\{0\}$,

$$
B(n)=\sum_{k=1}^{n}\left(\begin{array}{l}
n \\
k
\end{array}\right) B(n-k)=\sum_{k=1}^{n} \frac{n !}{k !(n-k) !} B(n-k) .
$$


Using this recursion formula, it can be verified that $B(1)=1, B(2)=3, B(3)=13$, $B(4)=75, B(5)=541, B(6)=4683$, etc.

We now return to the main issue addressed in this paper - a class of rank-mobility indices that are based on the Kemeny measure. The class can be expressed in terms of a one-parameter family of indices defined as follows. For all $c \in \mathbb{R}_{++}$, for all $N \in \mathcal{N}$ and for all $\left(R^{0}, R^{1}\right) \in \mathcal{R}^{N} \times \mathcal{R}^{N}$,

$$
M_{K}^{c}\left(R^{0}, R^{1}\right)=\frac{c}{n^{2}} M_{K}\left(R^{0}, R^{1}\right)=\frac{c}{n^{2}}\left(\left|R^{0} \backslash R^{1}\right|+\left|R^{1} \backslash R^{0}\right|\right) .
$$

The multiplicative constant $c / n^{2}$ reflects the influence of our replication-invariance property, to be defined shortly. Note that the relative mobility of any two pairs of social-status rankings does not depend on the specific (positive) value of the parameter $c$ - clearly,

$$
\begin{aligned}
& M_{K}^{c}\left(R^{0}, R^{1}\right)=\frac{c}{n^{2}} M_{K}\left(R^{0}, R^{1}\right) \geq \frac{c}{\bar{n}^{2}} M_{K}\left(\bar{R}^{0}, \bar{R}^{1}\right)=M_{K}^{c}\left(\bar{R}^{0}, \bar{R}^{1}\right) \Leftrightarrow \\
& M_{K}^{c^{\prime}}\left(R^{0}, R^{1}\right)=\frac{c^{\prime}}{n^{2}} M_{K}\left(R^{0}, R^{1}\right) \geq \frac{c^{\prime}}{\bar{n}^{2}} M_{K}\left(\bar{R}^{0}, \bar{R}^{1}\right)=M_{K}^{c^{\prime}}\left(\bar{R}^{0}, \bar{R}^{1}\right)
\end{aligned}
$$

for all $c, c^{\prime} \in \mathbb{R}_{++}$, for all $N, \bar{N} \in \mathcal{N}$, for all $\left(R^{0}, R^{1}\right) \in \mathcal{R}^{N} \times \mathcal{R}^{N}$ and for all $\left(\bar{R}^{0}, \bar{R}^{1}\right) \in$ $\mathcal{R}^{\bar{N}} \times \mathcal{R}^{\bar{N}}$ where $n$ and $\bar{n}$ correspond to the cardinalities of $N$ and $\bar{N}$ respectively.

We now introduce the axioms that are used in our characterization of the generalized Kemeny mobility measures. The first of these appears to be very non-controversial. It demands that the rank-mobility measure $M$ assumes a value of zero if there is no movement at all-that is, if all relative status ranks are the same in period zero and in period one. Furthermore, rank mobility is assumed to be positive whenever there is a change in relative ranks.

Zero at identity only. For all $N \in \mathcal{N}$ and for all $\left(R^{0}, R^{1}\right) \in \mathcal{R}^{N} \times \mathcal{R}^{N}$

$$
M\left(R^{0}, R^{1}\right)=0 \Leftrightarrow R^{0}=R^{1}
$$

The next axiom guarantees that mobility depends on the aggregate movement across social-status ranks only, in the sense that the measure should not discriminate between a move from a ranking $R^{0}$ to a ranking $R^{1}$ and the reverse move from $R^{1}$ to $R^{0}$. Note that this is a plausible property because we are measuring overall mobility (in the sense of movements in relative ranks) as opposed to phenomena such as upwards mobility; see, for instance, Schluter and Van de gaer (2011).

Symmetry. For all $N \in \mathcal{N}$ and for all $\left(R^{0}, R^{1}\right) \in \mathcal{R}^{N} \times \mathcal{R}^{N}$,

$$
M\left(R^{1}, R^{0}\right)=M\left(R^{0}, R^{1}\right) .
$$

In addition to having an appealing geometric interpretation, the well-known triangle inequality is a natural requirement in the context of rank-mobility measurement. The triangle inequality ensures that a direct move from a social-status ranking $R^{0}$ to a ranking 
$R^{2}$ cannot be associated with a higher degree of mobility in comparison to a move from $R^{0}$ to $R^{2}$ that involves a "detour" via a ranking $R^{1}$.

Triangle inequality. For all $N \in \mathcal{N}$ and for all $R^{0}, R^{1}, R^{2} \in \mathcal{R}^{N}$,

$$
M\left(R^{0}, R^{2}\right) \leq M\left(R^{0}, R^{1}\right)+M\left(R^{1}, R^{2}\right)
$$

Yet another extremely plausible property of a rank-mobility measure is that the individuals in a society be treated anonymously, paying no attention to their identities. Thus, we require that permuting the labels that we assign to the members of a society does not affect the value of our measure. In order to express this as a formal axiom, we require some further notation. For any $N \in \mathcal{N}$, let $\pi: N \rightarrow N$ be a bijective function. For $R \in \mathcal{R}^{N}$, we define the relation $R_{\pi}$ by letting, for all $a, b \in N$,

$$
(\pi(a), \pi(b)) \in R_{\pi} \Leftrightarrow(a, b) \in R .
$$

Thus, the mapping $\pi$ permutes the labels of the agents by assigning the label $\pi(a)$ to the individual that was previously labeled individual $a \in N$. Anonymity demands that the mobility measure is invariant with respect to such permutations.

Anonymity. For all $N \in \mathcal{N}$, for all $\left(R^{0}, R^{1}\right) \in \mathcal{R}^{N} \times \mathcal{R}^{N}$ and for all permutations $\pi: N \rightarrow N$,

$$
M\left(R_{\pi}^{0}, R_{\pi}^{1}\right)=M\left(R^{0}, R^{1}\right) .
$$

The only axiom that may be considered to be controversial to some extent is an additivity property. We are well aware of this observation but it seems to us that it is not too hard to argue that it nevertheless is of some strong appeal; after all, various forms of additivity are ubiquitous in the theory of social index numbers and the separability assumptions that typically underly these assumptions have a solid foundation. In our context, this is where the notion of an ordering being located "between" two orderings comes into play. An ordering $R^{1} \in \mathcal{R}^{N}$ is between $R^{0} \in \mathcal{R}^{N}$ and $R^{2} \in \mathcal{R}^{N}$ if

$$
R^{0} \cap R^{2} \subseteq R^{1} \subseteq R^{0} \cup R^{2}
$$

This seemingly abstract formulation has, as does the Kemeny measure, an intuitively appealing interpretation: an ordering $R^{1}$ is between two orderings $R^{0}$ and $R^{2}$ if the former lies on a shortest path from $R^{0}$ to $R^{2}$. For instance, returning to Figure 2, we see that (123) is between 123 and 321 but (123) is not between 123 and (13)2.

Additivity means that attention is paid to the notion of a shortest path between two social-status rankings. If an ordering $R^{1}$ lies on a shortest path between two orderings $R^{0}$ and $R^{2}$ (that is, $R^{1}$ is between $R^{0}$ and $R^{2}$ ), then the inequality in the triangle inequality should be satisfied with an equality. This ensures that due consideration is given to the defining property of a shortest path: if the additional step via $R^{1}$ when moving from $R^{0}$ to $R^{2}$ does not involve any "detour" in the sense of increasing the distance traveled from $R^{0}$ to $R^{2}$, this should not affect the value of the mobility measure. 
Additivity. For all $N \in \mathcal{N}$ and for all $R^{0}, R^{1}, R^{2} \in \mathcal{R}^{N}$, if $R^{1}$ is between $R^{0}$ and $R^{2}$, then

$$
M\left(R^{0}, R^{2}\right)=M\left(R^{0}, R^{1}\right)+M\left(R^{1}, R^{2}\right) .
$$

The conjunction of zero at identity only, symmetry and additivity implies the triangle inequality. This is an immediate consequence of Theorem 3 of Can and Storcken (2015).

Lemma 1. If a rank-mobility measure $M$ satisfies zero at identity only, symmetry and additivity, then $M$ satisfies the triangle inequality.

All of the axioms introduced so far only impose restrictions on fixed-population-size mobility comparisons. Our final property provides a link between comparisons across societies with different population sizes. Clearly, the possibility of making such comparisons is essential in order for the resulting measures to be applicable in comparisons across different societies, which cannot reasonably be assumed to have the same population size. A popular requirement that can be found in virtually all problems involving social index numbers that are capable of performing variable-dimension comparisons is replication invariance. We first need some additional notation. Given any ranking $R \in \mathcal{R}^{N}$, we use the position $t$-tuple $I^{R}=\left(I_{1}^{R}, I_{2}^{R}, \ldots, I_{t}^{R}\right)$ to denote the partitioning of the agents in $N$ into $t \in \mathbb{N}$ indifference classes according to each individual's position in the ranking $R$. For instance for $R=12(34) 5$, we obtain $I^{R}=(\{1\},\{2\},\{3,4\},\{5\})$ with the obvious interpretation $I_{1}^{R}=\{1\}, I_{2}^{R}=\{2\}, I_{3}^{R}=\{3,4\}$ and $I_{4}^{R}=\{5\}$.

Consider two disjoint sets of agents $N, N_{1} \in \mathcal{N}$ with the same cardinality and any ranking $R \in \mathcal{R}^{N}$ with the position $t$-tuple $I^{R}$. Consider any bijection $\sigma_{1}: N \rightarrow N_{1}$. We denote a twofold replica of $R$ by $\sigma_{1} R \in \mathcal{R}^{N \cup N_{1}}$ whenever for all $j \in\{1, \ldots, t\}$, we have $I_{j}^{\sigma_{1} R}=I_{j}^{R} \cup \sigma_{1}\left(I_{j}^{R}\right)$ where $\sigma_{1}\left(I_{j}^{R}\right)=\left\{\sigma_{1}(a) \mid a \in I_{j}^{R}\right\}$. This notion of a twofold replica extends naturally to higher numbers. Consider any $k>2$ pairwise disjoint sets of agents $N, N_{1}, \ldots, N_{k-1} \in \mathcal{N}$ with the same cardinality. Consider any $k-1$ bijections $\sigma_{i}: N \rightarrow N_{i}$ for all $i \in\{1, \ldots, k-1\}$. We denote a $k$-fold replica of $R$ by $\sigma_{1} \ldots \sigma_{k-1} R \in \mathcal{R}^{N \cup N_{1} \cup \ldots \cup N_{k-1}}$ whenever for all $j \in\{1, \ldots, t\}$ we have $I_{j}^{\sigma_{1} \ldots \sigma_{k-1} R}=I_{j}^{R} \cup \sigma_{1}\left(I_{j}^{R}\right) \cup \ldots \cup \sigma_{k-1}\left(I_{j}^{R}\right)$ where $\sigma_{i}\left(I_{j}^{R}\right)=\left\{\sigma_{i}(a) \mid a \in I_{j}^{R}\right\}$ for all $i \in\{1, \ldots, k-1\}$. If there is no ambiguity we also write $k R$ for a generic $k$-fold replica of $R$.

Consider any $k \geq 2$ pairwise disjoint sets of agents $N, N_{1}, \ldots, N_{k-1} \in \mathcal{N}$ with the same cardinality. Consider any $k-1$ bijections $\sigma_{i}: N \rightarrow N_{i}$ for all $i \in\{1, \ldots, k-1\}$. For any pair of rankings $\left(R^{0}, R^{1}\right) \in \mathcal{R}^{N} \times \mathcal{R}^{N}$, one can construct a $k$-fold replica of this pair, i.e., $\left(\sigma_{1} \ldots \sigma_{k-1} R^{0}, \sigma_{1} \ldots \sigma_{k-1} R^{1}\right)$, in the aforementioned way. Again, if there is no ambiguity we also write $\left(k R_{0}, k R^{1}\right)$ for a generic $k$-fold replica of this pair. A $k$-fold replica simply expands the indifference classes of each ranking by adding $k-1$ agents to each of them correspondingly. Replication invariance requires that the mobility associated with this replicated pair is the same as the mobility assigned to the original pair. Hence the mobility measure is insensitive to replications of the pairs of rankings under consideration.

Replication invariance. For all $N \in \mathcal{N}$, for all $\left(R^{0}, R^{1}\right) \in \mathcal{R}^{N} \times \mathcal{R}^{N}$ and for all $k \in$ $\mathbb{N} \backslash\{0,1\}$,

$$
M\left(k R^{0}, k R^{1}\right)=M\left(R^{0}, R^{1}\right) .
$$


We first illustrate the idea of replication invariance by an example. Then we show that the axiom implies anonymity.

Example 1. Consider the following sets of agents $N=\{1,2,3\}, N_{1}=\{4,5,6\}$ and $N_{2}=$ $\{7,8,9\}$. Let the orderings $R^{0}, R^{1} \in \mathcal{R}^{N}$ be such that $R^{0}=123$ and $R^{1}=312$ and consider two bijections $\sigma_{1}: N \rightarrow N_{1}$ (where 1,2,3 are bijected to 4, 5, 6 respectively) and $\sigma_{2}: N \rightarrow N_{2}$ (where $1,2,3$ are bijected to $7,8,9$ respectively). Then the pair of rankings $\left(\bar{R}^{0}, \bar{R}^{1}\right) \in$ $\mathcal{R}^{N \cup N_{1} \cup N_{2}} \times \mathcal{R}^{N \cup N_{1} \cup N_{2}}$ where $\bar{R}^{0}=(\mathbf{1 4 7})\left(\mathbf{2 5 8 ) ( 3 6 9 )}\right.$ and $\bar{R}^{1}=(\mathbf{3 6 9})(\mathbf{1 4 7})(\mathbf{2 5 8 )}$ is a 3-fold replica of the pair $\left(R^{0}, R^{1}\right)$. Therefore replication invariance requires that $M\left(\bar{R}^{0}, \bar{R}^{1}\right)=$ $M\left(R^{0}, R^{1}\right)$.

Lemma 2. If a rank-mobility measure $M$ satisfies replication invariance, then $M$ satisfies anonymity.

Proof. Let $M$ satisfy replication invariance and consider any $N \in \mathcal{N}$, any $\left(R^{0}, R^{1}\right) \in$ $\mathcal{R}^{N} \times \mathcal{R}^{N}$ and any permutation $\pi: N \rightarrow N$. We need to show that $M\left(R_{\pi}^{0}, R_{\pi}^{1}\right)=M\left(R^{0}, R^{1}\right)$. Consider any set of agents $\bar{N} \in \mathcal{N}$ with the same cardinality as $N$ such that $\bar{N} \cap N=\emptyset$.

Take any bijection $\sigma_{1}: N \rightarrow \bar{N}$ and let $\bar{R}^{0}$ and $\bar{R}^{1} \in \mathcal{R}^{\bar{N}}$ denote the rankings which are formed by relabeling $R^{0}, R^{1} \in \mathcal{R}^{N}$ according to $\sigma_{1}$, that is, $I^{\bar{R}^{0}}=\sigma_{1}\left(I^{R^{0}}\right)$ and $I^{\bar{R}^{1}}=$ $\sigma_{1}\left(I^{R^{1}}\right)$. Let $\rho_{1}: N \rightarrow \bar{N}$ be the composite function $\sigma_{1} \circ \pi^{-1}$ where $\pi^{-1}$ is the inverse of the function $\pi$. Furthermore note that $\rho_{1}$ is also a bijection from $N$ to $\bar{N}$.

Note that $\left(\sigma_{1} R^{0}, \sigma_{1} R^{1}\right)$ is a 2 -fold replica of $\left(R^{0}, R^{1}\right)$, and $\left(\rho_{1} R_{\pi}^{0}, \rho_{1} R_{\pi}^{1}\right)$ is a 2 -fold replica of $\left(R_{\pi}^{0}, R_{\pi}^{1}\right)$. Hence, replication invariance implies

$$
M\left(\sigma_{1} R^{0}, \sigma_{1} R^{1}\right)=M\left(R^{0}, R^{1}\right)
$$

and

$$
M\left(\rho_{1} R_{\pi}^{0}, \rho_{1} R_{\pi}^{1}\right)=M\left(R_{\pi}^{0}, R_{\pi}^{1}\right)
$$

Note also that both $\left(\sigma_{1} R^{0}, \sigma_{1} R^{1}\right)$ and $M\left(\rho_{1} R_{\pi}^{0}, \rho_{1} R_{\pi}^{1}\right)$ can be rewritten as $\left(\sigma_{1}^{-1} \bar{R}^{0}, \sigma_{1}^{-1} \bar{R}^{1}\right)$ and $\left(\rho_{1}^{-1} \bar{R}^{0}, \rho_{1}^{-1} \bar{R}^{1}\right)$ respectively. Hence they both are 2-fold replicas of $\left(\bar{R}^{0}, \bar{R}^{1}\right)$ and, by replication invariance, it follows that

$$
M\left(\sigma_{1}^{-1} \bar{R}^{0}, \sigma_{1}^{-1} \bar{R}^{1}\right)=M\left(\rho_{1}^{-1} \bar{R}^{0}, \rho_{1}^{-1} \bar{R}^{1}\right)
$$

Using (1) and (2), we conclude that $M\left(R_{\pi}^{0}, R_{\pi}^{1}\right)=M\left(R^{0}, R^{1}\right)$.

\section{The generalized Kemeny rank-mobility measures}

In order to state and prove the characterization result alluded to above, we make use of a recent characterization of a class of generalized Kemeny distance functions in a fixedpopulation-size setting. This characterization is due to Can and Storcken (2013) and it represents an improvement on the traditional axiomatization of Kemeny and Snell (1962). Of course, we could alternatively invoke the original Kemeny-Snell characterization but, because their result involves a redundant axiom, it clearly is preferable to employ the 
tighter characterization theorem established by Can and Storcken (2013). Among other results, Can and Storcken (2013) proved the following theorem that, for convenience, we state in the context of rank-mobility measurement. Because of Lemma 1, which follows from Theorem 3 in Can and Storcken (2015), we can drop the triangle inequality from their version. Note that this leaves the independence of their axioms intact because they formulated the properties of a metric (including non-negativity which is built into our definition of a rank-mobility measure) as a single axiom.

Theorem 1. A rank-mobility measure $M$ satisfies zero at identity only, symmetry, anonymity and additivity if and only if there exists a set $\left\{c_{n} \in \mathbb{R}_{++} \mid N \in \mathcal{N}\right\}$ such that, for all $N \in \mathcal{N}$ and for all $\left(R^{0}, R^{1}\right) \in \mathcal{R}^{N} \times \mathcal{R}^{N}$,

$$
M\left(R^{0}, R^{1}\right)=c_{n} M_{K}\left(R^{0}, R^{1}\right)=c_{n}\left(\left|R^{0} \backslash R^{1}\right|+\left|R^{1} \backslash R^{0}\right|\right) .
$$

Notice that the parameter $c_{n}$ depends only on the size of the society $N$ in question, and is the same for any equally large society. Theorem 1, the proof of which can be obtained by applying the requisite results in Can and Storcken (2013, 2015), improves upon the corresponding result reported in Kemeny (1959) and Kemeny and Snell (1962). These earlier authors employ, in addition to the axioms in the above theorem, a reducibility property that applies across different population sizes. Loosely speaking, reducibility requires that the distance between two orderings is unchanged if individuals are removed that are ranked identically at the top or at the bottom of the two orderings; see Can and Storcken (2013) for details. Can and Storcken (2013) show that reducibility is redundant in the Kemeny and Snell (1962) characterization of the Kemeny measure and, as a consequence, come up with a characterization that is formally and conceptually stronger than the original axiomatization. In addition to establishing that the axioms employed in the original characterization are not independent, they are able to drop the only axiom that (at least implicitly) imposes restrictions on variable-population-size comparisons. Although we define the notion of a distance function and the axioms in a variable-population-size setting, it is clear that fixed-size versions of the above result can be formulated in a straightforward manner because each of the properties used only applies to orderings involving the same population size. In contrast, Kemeny and Snell's (1962) reducibility axiom cannot even be defined in a fixed-size framework and, thus, they did not succeed in providing a characterization on the domain for which Can and Storcken's (2013) results are valid.

We can now present our main result which is a characterization of the class of generalized Kemeny mobility measures. We do so by strengthening anonymity to replication invariance.

Theorem 2. A rank-mobility measure $M$ satisfies zero at identity only, symmetry, additivity and replication invariance if and only if there exists $c \in \mathbb{R}_{++}$such that, for all $N \in \mathcal{N}$ and for all $\left(R^{0}, R^{1}\right) \in \mathcal{R}^{N} \times \mathcal{R}^{N}$,

$$
M\left(R^{0}, R^{1}\right)=M_{K}^{c}\left(R^{0}, R^{1}\right)=\frac{c}{n^{2}} M_{K}\left(R^{0}, R^{1}\right)=\frac{c}{n^{2}}\left(\left|R^{0} \backslash R^{1}\right|+\left|R^{1} \backslash R^{0}\right|\right) .
$$


Proof. The 'if' part of the theorem statement is straightforward to verify. To prove the 'only-if' part, suppose that the axioms of the theorem statement are satisfied. By Lemmas 1 and 2 , we can invoke Theorem 1 to conclude that there exists a set $\left\{c_{n} \in \mathbb{R}_{++} \mid N \in \mathcal{N}\right\}$ such that, for all $N \in \mathcal{N}$ and for all $\left(R^{0}, R^{1}\right) \in \mathcal{R}^{N} \times \mathcal{R}^{N}$,

$$
M\left(R^{0}, R^{1}\right)=c_{n} M_{K}\left(R^{0}, R^{1}\right)=c_{n}\left(\left|R^{0} \backslash R^{1}\right|+\left|R^{1} \backslash R^{0}\right|\right) .
$$

Take any $\left(R^{0}, R^{1}\right) \in \mathcal{R}^{N} \times \mathcal{R}^{N}$ such that $R^{0} \neq R^{1}$. Consider any $k$-fold replica of this pair, and denote it by $\left(k R^{0}, k R^{1}\right) \in \mathcal{R}^{\bar{N}} \times \mathcal{R}^{\bar{N}}$ where $\bar{N}=N \cup N_{1} \cup \ldots N_{k-1}$ as in the definition of a $k$-fold replica. Then there exists a sequence of bijections $\kappa=\left(\sigma_{1}, \ldots, \sigma_{k-1}\right)$ such that, for all $a, b \in N$ and for all $i, j \in\{1, \ldots, k-1\}$, we have

$$
\left(\sigma_{i}(a), \sigma_{j}(b)\right) \in k R^{0} \Leftrightarrow(a, b) \in R^{0}
$$

and

$$
\left(\sigma_{i}(a), \sigma_{j}(b)\right) \in k R^{1} \Leftrightarrow(a, b) \in R^{1} .
$$

Then for any $(a, b) \in R^{0} \backslash R^{1}$, we have

$$
\begin{array}{ccccc}
(a, b), & \left(a, \sigma_{1}(b)\right), & \ldots, & \left(a, \sigma_{k-1}(b)\right) & \in k R^{0} \backslash k R^{1}, \\
\left(\sigma_{1}(a), b\right), & \left(\sigma_{1}(a), \sigma_{1}(b)\right), & \ldots, & \left(\sigma_{1}(a), \sigma_{k-1}(b)\right) & \in k R^{0} \backslash k R^{1}, \\
\vdots & & & \\
\left(\sigma_{k-1}(a), b\right), \quad\left(\sigma_{k-1}(a), \sigma_{1}(b)\right), & \ldots, & \left(\sigma_{k-1}(a), \sigma_{k-1}(b)\right) & \in k R^{0} \backslash k R^{1}
\end{array}
$$

Thus, for each pair in $R^{0} \backslash R^{1}$, there are $k^{2}$ pairs in $k R^{0} \backslash k R^{1}$ and, analogously, for each pair in $R^{1} \backslash R^{0}$, there are $k^{2}$ pairs in $k R^{1} \backslash k R^{0}$. Hence,

$$
\left|k R^{0} \backslash k R^{1}\right|+\left|k R^{1} \backslash k R^{0}\right|=k^{2}\left(\left|R^{0} \backslash R^{1}\right|+\left|R^{1} \backslash R^{0}\right|\right)=k^{2} M_{K}\left(R^{0}, R^{1}\right) .
$$

Note that $c_{n}$ is defined on the basis of cardinalities of the societies. As $|\bar{N}|=n k$ we can simply use the $c_{n k}$ notation for the $k$-fold replica. Using (3), we obtain

$$
\begin{aligned}
M\left(k R^{0}, k R^{1}\right) & =c_{n k}\left(\left|k R^{0} \backslash k R^{1}\right|+\left|k R^{1} \backslash k R^{0}\right|\right) \\
& =c_{n k} k^{2} M_{K}\left(R^{0}, R^{1}\right)
\end{aligned}
$$

and

$$
M\left(R^{0}, R^{1}\right)=c_{n} M_{K}\left(R^{0}, R^{1}\right) .
$$

Therefore, replication invariance requires that, for all $n, k \in \mathbb{N} \backslash\{0,1\}$,

$$
c_{n k} k^{2} M_{K}\left(R^{0}, R^{1}\right)=c_{n} M_{K}\left(R^{0}, R^{1}\right) .
$$

Because $R^{0} \neq R^{1}, M_{K}\left(R^{0}, R^{1}\right)>0$ and, thus, (4) demands that

$$
c_{n k} k^{2}=c_{n} \quad \text { for all } n, k \in \mathbb{N} \backslash\{0,1\} .
$$


Setting $n=2$ in (5), we obtain

$$
c_{2 k}=\frac{c_{2}}{k^{2}} \quad \text { for all } k \in \mathbb{N} \backslash\{0,1\}
$$

and, letting $m=2 k$ and defining $c=4 c_{2} \in \mathbb{R}_{++}$, (6) can be rewritten as

$$
c_{m}=\frac{c_{2}}{(m / 2)^{2}}=\frac{4 c_{2}}{m^{2}}=\frac{c}{m^{2}} \quad \text { for all even } m \in \mathbb{N} \backslash\{0,1\} .
$$

Now let $n$ be odd. Let $k=2$ in (5), which implies that $n k=2 n$. Substituting into (6), it follows that

$$
4 c_{2 n}=c_{n}
$$

and, because $2 n$ is even, (7) implies

$$
c_{2 n}=\frac{c}{4 n^{2}} .
$$

Combining (8) and (9), it follows that

$$
c_{n}=\frac{c}{n^{2}} \quad \text { for all odd } n \in \mathbb{N} \backslash\{0,1\}
$$

and, together with (7),

$$
c_{n}=\frac{c}{n^{2}} \quad \text { for all } n \in \mathbb{N} \backslash\{0,1\} .
$$

Substituting back into (3) yields the desired result.

The axioms employed in Theorem 2 are independent, as established by means of the following examples.

Define, for all $N \in \mathcal{N}$ and for all $\left(R^{0}, R^{1}\right) \in \mathcal{R}^{N} \times \mathcal{R}^{N}$,

$$
M\left(R^{0}, R^{1}\right)=0 .
$$

Clearly, this rank-mobility measure satisfies symmetry, additivity and replication invariance but it fails to satisfy zero at identity only.

Let, for all $N \in \mathcal{N}, I_{U}^{n}=N \times N$-that is, $I_{U}^{n}$ is the universal indifference relation. Now define, for all $N \in \mathcal{N}$ and for all $\left(R^{0}, R^{1}\right) \in \mathcal{R}^{N} \times \mathcal{R}^{N}$,

$$
M\left(R^{0}, R^{1}\right)= \begin{cases}\frac{1}{n^{2}} M_{K}\left(R^{0}, R^{1}\right)+1 & \text { if } R^{0} \neq I_{U}^{n} \text { and } I_{U}^{n} \text { is between } R^{0} \text { and } R^{1}, \\ \frac{1}{n^{2}} M_{K}\left(R^{0}, R^{1}\right) & \text { otherwise. }\end{cases}
$$

This rank-mobility measure satisfies zero at identity only, additivity and replication invariance but it violates symmetry.

Let, for all $N \in \mathcal{N}$ and for all $\left(R^{0}, R^{1}\right) \in \mathcal{R}^{N} \times \mathcal{R}^{N}$,

$$
M\left(R^{0}, R^{1}\right)= \begin{cases}0 & \text { if } R^{0}=R^{1} \\ \frac{1}{n^{2}} & \text { if } R^{0} \neq R^{1}\end{cases}
$$

This rank-mobility measure satisfies zero at identity only, symmetry and replication invariance but it violates additivity.

Finally, the Kemeny measure $M_{K}$ satisfies zero at identity only, symmetry and additivity but it does not satisfy replication invariance. 


\section{Concluding remarks}

The contribution of this paper consists primarily of an alternative approach to the measurement of rank mobility as initiated by D'Agostino and Dardanoni (2009). While the notion of rank mobility seems to rest on a solid conceptual foundation, it is clear that there are shortcomings as well (as is the case for all areas of economic measurement in which no consensus has been reached yet as far as the existence of a single superior index - or class of indices - is concerned). We propose a class of replication-invariant mobility indices that generalize one of the two most fundamental measures of non-parametric rank correlation - namely, Kendall's (1938) $\tau$ index. The rank-mobility preorders D'Agostino and Dardanoni (2009) characterize are linked to the other common measure of correlation, which is Spearman's (1904) $\rho$ index. Thus, our analysis is by no means intended to diminish the importance of alternative suggestions that have appeared in the literature. When added to D'Agostino's and Dardanoni's (2009) fundamental contribution, the work reported here may be viewed as providing an additional argument in favor of the further exploration of rank-based mobility measurement.

It is possible to narrow down our class of mobility measures by imposing a normalization property that forces the value of the parameter $c$ to be equal to one, just as the Kemeny measure can be obtained by adding a normalization requirement so that each of the parameters $c_{n}$ in Can and Storcken's (2013) result must assume a value of one; see Can and Storcken (2013) for details. We refrain from providing a formal statement of this observation because it is relatively straightforward and does not add all that much if an ordinal interpretation is given to the mobility measures. In contrast, the normalization property is of more value in the Can-Storcken result because, without such a property, variable-population-size comparisons are not restricted in any way.

Without going into any technical details, we note that the rank-based setting can be applied to the measurement of income mobility in a straightforward manner. All that is required is the addition of an axiom that ensures that only social-status ranks matter and, furthermore, slight modifications of zero at identity only and the additivity axiom so as to take into account this ranks-only property.

\section{References}

Can, B. and T. Storcken (2013), A re-characterization of the Kemeny distance, Maastricht University School of Business and Economics, RM/13/009.

Can, B. and T. Storcken (2015), Comparing orders, rankings, queues, tournaments and lists, Maastricht University School of Business and Economics, RM/15/020.

Cowell, F.A. and E. Flachaire (2011), Measuring mobility, GREQAM Working Paper 2011-21.

D'Agostino, M. and V. Dardanoni (2009), The measurement of rank mobility, Journal of Economic Theory 144, 1783-1803. 
Dickens, R. (1999), Caught in a trap? Wage mobility in Great Britain: 1975-1994, Economica 67, 477-497.

Fields, G.S. (2008), Income mobility, in: S.N. Durlauf and L.E. Blume (eds.), The New Palgrave Dictionary of Economics Online, Palgrave Macmillan, New York. Available at http://www.dictionaryofeconomics.com/article?id=pde2008_I000271 doi:10.1057/9780230226203.0770

Fields, G.S. and E.A. Ok (1999), The measurement of income mobility: an introduction to the literature, in: J. Silber (ed.), Handbook of Income Inequality Measurement, Kluwer Academic Publishers, Norwell, pp. 557-596.

Jäntti, M. and S.R. Jenkins (2014), Income mobility, in: A.B. Atkinson and F. Bourguignon (eds.), Handbook of Income Distribution, Vol. 2, Elsevier, Amsterdam, pp. 807-936.

Kemeny, J.G. (1959), Mathematics without numbers, Daedalus 88, 577-591.

Kemeny, J.G. and J.L. Snell (1962), Preference rankings: an axiomatic approach, in: J.G. Kemeny and J.L. Snell (eds.), Mathematical Models in the Social Sciences, Blaisdell Publishing Company, New York, pp. 9-23.

Kendall, M.G. (1938), A new measure of rank correlation, Biometrika 30, 81-93.

Maasoumi, E. (1998), On mobility, in: D. Giles and A. Ullah (eds.), Handbook of Applied Economic Statistics, Marcel Dekker, New York, pp. 119-175.

Schluter, C. and D. Van de gaer (2011), Upward structural mobility, exchange mobility, and subgroup consistent mobility measurement: U.S.-German mobility rankings revisited, Review of Income and Wealth 57, 1-22.

Spearman, C. (1904), The proof and measurement of association between two things, American Journal of Psychology 15, 72-101. 